\title{
Impact of COVID-19 in the Mental Health in Elderly: Psychological and Biological Updates
}

\author{
Roberta Eduarda Grolli ${ }^{1,2}$. Maiqueli Eduarda Dama Mingoti ${ }^{1,2} \cdot$ Amanda Gollo Bertollo ${ }^{1,2}$. \\ Adriana Remião Luzardo ${ }^{1,3}$ • João Quevedo ${ }^{4,5,6,7}$ • Gislaine Zilli Réus ${ }^{4}$ - Zuleide Maria Ignácio ${ }^{1,2,4}$ (D)
}

Received: 4 August 2020 / Accepted: 4 December 2020 / Published online: 6 January 2021

(C) The Author(s), under exclusive licence to Springer Science+Business Media, LLC part of Springer Nature 2021

\begin{abstract}
Since December 2019, the world has been experiencing the challenge of facing coronavirus disease-19 (COVID-19), a severe infectious disease caused by the new coronavirus, SARS-CoV-2. The individuals with the most severe symptoms and the highest risk of death are the elderly and those with chronic illness. Among chronic conditions, those with a certain degree of chronic inflammation may predispose to a more severe evolution of COVID-19. Elderly with psychiatric disorders can present a persistent inflammatory state, a characteristic of the age's immunological senescence, but the disorder can accentuate that. Social isolation is still the safest way to avoid contamination. However, isolated older people may have or worsen mental health conditions due to isolation and health concerns. In this scenario, a SARS-CoV-2 infection may progress to more severe disease. Conversely, COVID-19 can predispose or aggravate psychiatric disorders, as it induces a cytokine storm, causing systemic hyper inflammation. It may damage the blood-brain barrier, resulting in inflammation in the central nervous system. Besides, SARS$\mathrm{CoV}-2$ is likely to reach and trigger an inflammatory process directly in the nervous system. This review makes an update about research on the mental health of the elderly during the pandemic. Also, it discusses the vulnerability of these individuals in the face of stress and in the case of contracting COVID-19, considering mainly the stress's hormonal and inflammatory mechanisms. Finally, the review points out possible care and attention strategies and entertainment and activities that can reduce the damage to mental and physical health and improve the elderly's quality of life.
\end{abstract}

Keywords COVID-19 $\cdot$ Elderly $\cdot$ Mental health $\cdot$ Psychiatric disorders $\cdot$ Inflammation

\section{Highlights}

- Isolation and concerns about COVID-19 may harm elderly mental health.

- Immunosenescence and pandemic stress increase the risk of psychiatric disorders.

- Stress and disorders may potentiate the elderly's inflammation and COVID-19 symptoms.

- SARS-CoV-2 hyperinflammation is a risk factor for elderly psychiatric disorders.

Zuleide Maria Ignácio

zuleideignacio@gmail.com

1 Undergraduate Nursing Course, Federal University of the Southern Frontier, Chapeco, SC, Brazil

2 Laboratory of Physiology, Pharmacology, Psychopathology, Postgraduate Program in Biomedical Sciences, Federal University of Southern Frontier, Chapeco, SC, Brazil

3 Laboratory of Innovation and Health Technologies, Federal University of the Southern Frontier, Chapeco, SC, Brazil

4 Laboratory of Translational Psychiatry, Graduate Program in Health Sciences, University of Southern Santa Catarina, Criciuma, SC, Brazil
5 Center of Excellence on Mood Disorders, Faillace Department of Psychiatry and Behavioral Sciences, McGovern Medical School, The University of Texas Health Science Center at Houston (UTHealth), Houston, TX, USA

6 Translational Psychiatry Program, Faillace Department of Psychiatry and Behavioral Sciences, McGovern Medical School, The University of Texas Health Science Center at Houston (UTHealth), Houston, TX, USA

7 Neuroscience Graduate Program, Graduate School of Biomedical Sciences, The University of Texas Health Science Center at Houston (UTHealth), Houston, TX, USA 


\section{Introduction}

A new coronavirus has spread globally since the first recorded human infections noted in December 2019. The virus was identified as the coronavirus of severe acute respiratory syndrome 2 (SARS-CoV-2) [1]. In early 2020, the coronavirus disease-19 (COVID-19) took on global proportions, and in March, the World Health Organization declared a state of a pandemic [2]. The mortality rate is around 2 to $3 \%$ for healthy adults. However, for the elderly, the propensity is three times higher [3]. Studies suggest that the elderly population may be more severely affected by COVID-19 infections because the virus can worsen symptoms from pre-existing conditions/ diseases [4]. In addition to the risks involving frequent comorbidities in the elderly, such as diabetes and hypertension, psychiatric disorders can be precipitated and accentuated and can increase the severity of COVID-19.

Still, without palliative or preventive treatment, it is essential to isolate patients, minimize face-to-face relationships, and perform quarantine. These actions imply a drastic change in routine. Consequently, waves of anxiety and insecurity have affected the elderly, leaving them susceptible to disorders' onset or aggravation during this period [5].

More than $20 \%$ of the world population over 60 years lives with a psychiatric disorder or neurological disease. Dementia, major depressive disorder (MDD), and anxiety are the most prevalent [6].

Some researchers argue that the lack of awareness and cognitive impairment, associated with stress, are factors that make this population more susceptible to infection $[4,5,7]$ and death due to environmental impoverishment and absence of stimuli [8]. Meanwhile, there is a silent fear feeling, which affects mainly those who already have a mental vulnerability. Once infected, these people will still have to confront stigmas related to their mental state in health institutions [9].

Although social distancing is an essential preventative mechanism, it promotes feelings of loneliness, influencing the onset of or increasing the vulnerability for those who already have a psychiatric disorder(s) [3]. In particular, the elderly form one of the most vulnerable groups. Age, dementia, and MDD are risk factors for suicide [4]. Comprehensive monitoring of this population is needed because the virus, and the stressors associated with social isolation can exacerbate the symptoms of age-associated disorders [10].

This review encompasses the increased vulnerabilities of the elderly population when exposed to COVID-19. Besides, COVID-19 exposure and the relationship between psychiatric disorders and stressors inherent in the elderly population (including environmental, psychological, and social) have been examined. Relevant pathophysiological characteristics underlying the situation of psychiatric disorders, which can be aggravated or worsened by a SARS-CoV-2 infection, are also reviewed. Biological mechanisms such as changes in the immune system, inflammation, and the relationship with the hypothalamus-pituitary-adrenal (HPA) axis are discussed. Emphasis has been placed upon the involvement of mechanism(s) in conditions related to advanced age, mental disorders, stress, and infectious disease, such as COVID-19.

\section{About COVID-19}

In December 2019 were recorded the first cases of coronavirus in humans. Wuhan, a metropolitan region in the Chinese province of Hubei, was the pandemic's initial focus. Current data account for $68,845,368$ diagnosed cases and 1,570,304 deaths worldwide. The numbers continue to increase exponentially [11]. Severe acute respiratory syndrome (SARS), identified 18 years ago, was associated with coronavirus existing in animals [12]. Complete genomic sequences were performed in 5 patients in the pandemic's initial phase, identifying that COVID-19 is $96 \%$ identical to the bat coronavirus genes. Human infection typically presents in alveolar cells. The virus uses angiotensin-converting enzyme 2 (ACE2) and replicates in the upper airways, facilitating transmission [13]. In addition to inducing viral pneumonia, SARS-CoV-2 can affect other organs. Main complications can include severe myocardial changes, arrhythmias, and shocks, especially in elderly individuals [14]. In addition to the high possibility of affecting crucial organic functions, the prevalence and risk of death increases in the elderly with chronic medical comorbidities, such as type 2 diabetes mellitus, hypertension, cerebral and vascular heart diseases, and obstructive pulmonary diseases, among others $[15,16]$.

In Italy, 9 out of 10 deaths occur in individuals aged 70 and over [4]. The mortality rate reached $29.6 \%$ for people over 80 years old [17]. In the USA, the elderly represent $80 \%$ of deaths due to COVID-19 [2]. Studies carried out in Wuhan, the pandemic's initial focus, showed higher mortality in those who had diabetes and cardiovascular diseases [18].

The leading cause of death is respiratory failure due to marked inflammation in the alveolar tissue. However, a physiological event called "cytokine storm" can cause a more severe symptom onset. Cytokine storm is characterized by the increased release of inflammatory cytokines, culminating in widespread and stringent inflammation in multiple tissues. This situation can lead to severe organ damage and death from multiple organ failure [19]. Viral SARS-CoV infection causes virus-linked pyroptosis, a form of cell death programmed from a potent inflammatory response [20]. The full inflammatory response in most patients is a physiological process that reduces viral load and culminates in the recovery of the infection. However, in a percentage of patients, the cytokines storm turns into a vicious cycle, increasing the release of various inflammatory cytokines, such as interleukins (IL) IL-1, IL-2, IL-6, IL-7, and tumor necrosis factor- $\alpha$ (TNF- $\alpha)$, among other 
inflammatory markers $[14,18,21]$. SARS-CoV-2 has been hypothesized to utilize the inflammatory response by antagonizing the interferon response, which increases viral replication. When inflammation rises, the infection becomes more severe. Immunosenescence can offer in the elderly and may be related to a low-grade inflammatory state (Fig. 1). The lowgrade inflammatory condition can be more frequent in older adults and comorbidity with other chronic diseases [19].

Some studies observed an increase in antibodies to other coronaviruses in older adults [22]. From this evidence, Zheng et al. [23] suggest that an increase in viral load and inflammatory processes can trigger the severity of COVID-19 in these individuals. These circulating antibodies would bind with a weak affinity to SARS-CoV-2, allowing the virus's entry into monocyte-macrophages through the Fc receptor and leading to an antibody-dependent infection enhancement. The lowaffinity binding to the antibody makes viral phagocytosis by lysosomes difficult, allowing an immune escape. Thus, the virus reproduces and is released by monocyte-macrophages along with a large amount of IL-6, TNF- $\alpha$, and other cytokines, culminating in a cytokine storm and $\mathrm{T}$ cells downregulation. This condition is related to the severity of COVID-19 and negatively correlated with pro-inflammatory cytokines in the individuals' serum [24].

On the other hand, the immunosenescence that occurs with increasing age seems to be related to a chronic low-grade inflammatory state (Fig. 1) [25], which can be a predisposing factor to aberrant inflammation, increasing the severity and risk of death in elderly [26].

\section{Anxiety and MDD: What Factors Are Predisposing During COVID-19?}

It is estimated that about 192 million people over the age of 60 have some mental or neurological disorder level. About $7 \%$ are affected by MDD and 3.8\% by anxiety. MDD and anxiety are the most prevalent disorders in this age group [6]. Some studies have observed that anxiety increases death's risk associated with several comorbidities in people over 75 years old [27].

Without vaccines or specific treatment for COVID-19, isolation has become the most efficient form of prevention against the contagion of SARS-CoV-2 [28]. However, prolonged periods can lead to feelings of loneliness and anger [29]. Doubts about the future are frequent, and expectations regarding their needs, associated with isolation, predispose them to stress. Together, these factors culminate in worsening psychiatric conditions of individuals who were, or were not, in professional care for depression and anxiety disorders $[3,30]$. As the disease progresses and the isolation continues, the threat of mental breakdowns becomes imminent [5].

Santini et al. [31] point to social disconnection as a catalyst for a negative spiral, which leads to seclusion. The symptoms

\section{STRESS AND IMMUNOSENESCENCE}

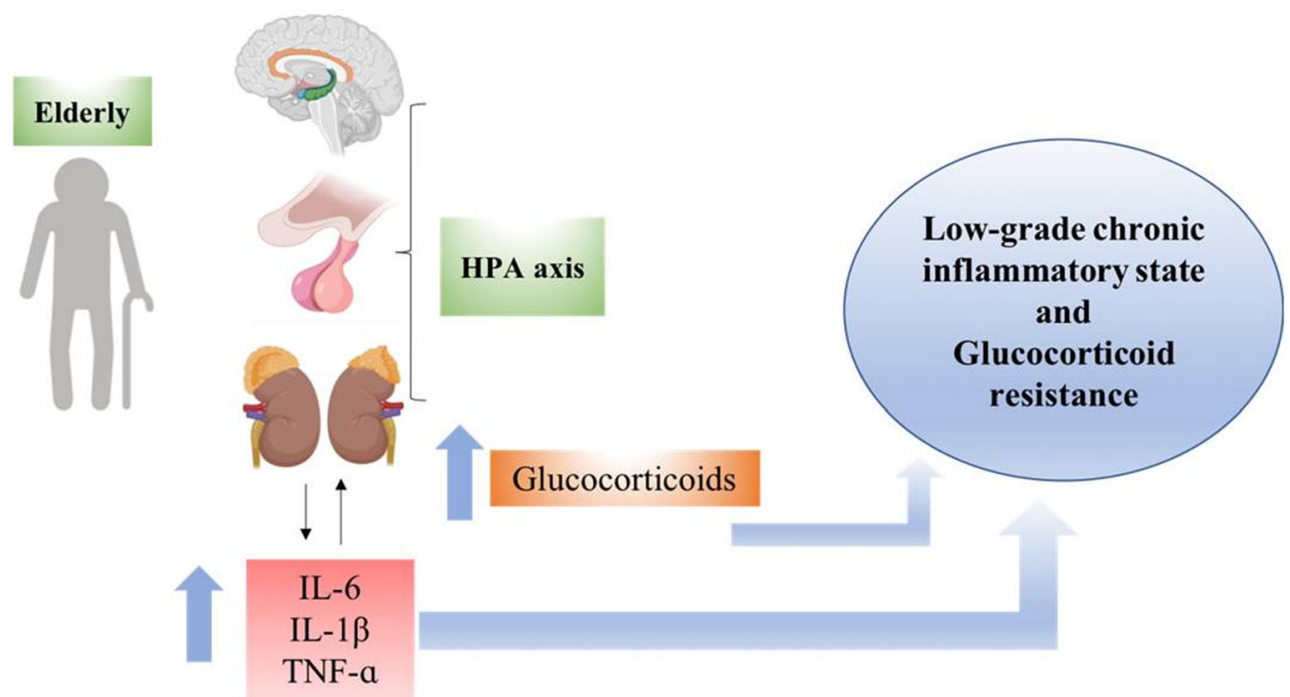

Fig. 1 The immunosenescence that occurs in aging is accompanied by an increase in pro-inflammatory cytokines, leading to a chronic inflammatory state. Chronic stress, caused by isolation and worries during the pandemic, can deregulate the negative feedback from the hypothalamus-pituitary-adrenal (HPA) axis, increasing the release of glucocorticoids by the adrenal glands. This condition is accompanied by an increase in cytokines, such as interleukin (IL) IL-1 $\beta$, IL-6, and tumor- necrosis factor- $\alpha$ (TNF- $\alpha$ ), among other pro-inflammatory molecules. In turn, pro-inflammatory cytokines contribute to the unbalance of the HPA axis, which may induce resistance to glucocorticoids, thus constituting a vicious cycle that contributes to the increase or maintenance of a chronic pro-inflammatory state. These associated conditions may culminate in a higher degree of chronic inflammation, predisposing the elderly to impair mental health. Images were extracted from Biorender app 
of some disorders may start or worsen, such as MDD and anxiety. A negative cycle occurs with social isolation, trapping the affected person within that spiral. Older people make up the most exposed group, especially those who live alone. If they stay in the negative spiral, they may have aggravated anxiety and depression symptoms, damaging their mental state.

Researchers have shown that MDD is related to many other chronic diseases, with the majority being more prominent in elderly individuals. Conversely, chronic diseases, such as diabetes, cardiovascular, and cerebrovascular diseases, are often accompanied by anxiety and MDD. These conditions raise the hypothesis of the etiopathogenesis of depression at the end of life $[30,32]$. The stressors that occur throughout life alter homeostasis in various physiological processes, such as oxidative stress, inflammation, and metabolic disorders, and culminate in an increase of allostatic load. This overload seems to be inherent in psychiatric disorders that frequently occur in comorbidities with frequent chronic diseases in old age [33].

Individuals with MDD and other disorders are prone to develop autoimmune and inflammatory diseases [33, 34]. Chronic inflammation can contribute to relevant neurotransmitter dysfunctions related to MDD and other psychiatric disorders [35], as well as impaired neurogenesis [36]. The increase in inflammation, which occurs with age and in psychiatric disorders, may be associated with other factors inherent to both conditions. This state of hyper inflammation can culminate in some degree of blood-brain barrier rupture. In these conditions, chronic microglial activation and neuroinflammation may occur [37]. Persistent microglial activation can lead to neuronal death and reduced neurogenesis [38].

Several studies have observed the involvement of inflammation in the beginning and in the maintenance of depression. Thus, researchers postulate the inflammatory hypothesis of depression [39]. The inflammation hypothesis considers that the inflammatory challenges in the CNS activate the microglia. The activated microglia releases several inflammatory cytokines, such as IL-1 $\beta$, IL-6, TNF- $\alpha$, IL-2, IL-18, and INF- $\gamma$. The cytokines activate the HPA axis and induce an increase in the indoleamine-2,3-dioxygenase (IDO) activity. In turn, IDO deviates tryptophan metabolism, reducing the synthesis of serotonin and liberating toxic metabolites. These metabolites alter the integrity of the blood-brain barrier and cause neurodegeneration and depression [39].

The routes by which inflammation can reach the CNS and contribute to trigger or worsen MDD include the following: cytokine trafficking through leaks in the blood-brain barrier; active transport through saturable molecules; activation of endothelial cells and perivascular macrophages, which secrete cytokines and other inflammatory mediators; binding to cytokine receptors present in afferent fibers of the vagus nerve; and allowing the sending of signals to specific regions, such as the nucleus of the solitary tract and hypothalamus. An important question concerning age is whether vascular insults at the end of life may have an essential role in bringing inflammatory cytokines to the CNS [40]. The nuclear factor kappa beta $(\mathrm{NF}-\mathrm{kB})$ appears to have a relevant role in communicating peripheral inflammation with the CNS [40].

Researchers point out that the mammalian target of rapamycin (mTOR) pathway is an important mechanism involved in MDD. The reduction of mTOR activity can lead to MDD, while drugs that increase the activity, such as ketamine, are considered potent antidepressants [41]. Some studies show that ketamine induces an anti-inflammatory profile through mTOR in parallel to antidepressant effects in humans' severe depression [42]. Notwithstanding, animal studies have shown that ketamine increased TNF- $\alpha$ in serum, and rapamycin did not reverse the effect of ketamine. Also, ketamine did not alter the levels of IL- $1 \beta$ and IL- 6 . Therefore, although the evidence is still controversial, it appears that the pro-inflammatory effect of ketamine in the study in question did not occur through the mTOR pathway [43]. Howsoever, some researches highlight the activity of the mTOR pathway on viruses because it is a target mechanism used by the virus, which triggers the rapid activation of machinery for the synthesis of the viral proteins, thus leading to an increased load and infection by SARSCoV-2, among other viruses. mTOR activity also acts in the pro-inflammatory process, as it induces inflammatory stimulation by IL-6 [23]. Besides, mTOR pathway activation seems to reduce the p53 protein, which is involved in apoptosis and reduction of viral replication [44]. Thus, the inhibition of the mTOR pathway could be a pharmacological strategy for reducing viral load and hyper inflammation [44, 45].

Some research also notes that severe treatment-resistant depression (TRD) is associated with an increase in several inflammatory proteins, such as IL-6, IL-8, and IL-12, among others [46]. The researchers also observed that older patients with a higher load of medication had higher inflammatory markers levels [46]. Previous studies have also noted that the increase in inflammatory molecules is also positively related to increasing age, among other situations of stress during life [47]. Considering that increasing age seems to be associated with immunosenescence, with a low-grade chronic inflammatory state [25], stress and psychiatric disorders can exacerbate inflammation, making the elderly more vulnerable to diseases that challenge the immune system, increasing inflammatory processes.

From another angle, the elevated release of glucocorticoids during chronic stress increases microglial activation and neuroinflammation [48]. Recent research also postulates that microglia's function is one of the critical mechanisms constituting the pathophysiology of depression, among other psychiatric disorders $[49,50]$. Repeated microglia activation can cause systemic inflammation and tissue damage. Besides, persistent inflammation can culminate in glucocorticoid resistance [51]. Thus, the relationship between the HPA axis and 
the immune system, considering stress and age, can trigger a vicious cycle, hyperactivating the endocrine and inflammatory mechanisms (Fig. 1), which make the elderly more susceptible, both to the worsening of COVID-19 symptoms and an increase in the severity of psychiatric disorders.

\section{Other Disorders-Predisposing Factors During COVID-19}

Other psychiatric and neurodegenerative disorders may predispose greater severity to the infectious condition of COVID19. In $11.9 \%$ of elderly Italian patients who died from Covid19, dementia was comorbidity [7]. Social exclusion due to isolation is one of the most considerable risks of cognitive impairment, increasing the risk of Alzheimer's disease [52]. Psychotic disorders [53] and obsessive and somatic symptoms related to the pandemic are also documented [54]. It is essential to consider that these diseases and post-traumatic stress disorder (PTSD) even present the deregulation of circulating pro-inflammatory cytokines. This event can aggravate the infectious condition [55-57].

Noteworthy are the studies on neuropsychiatric diseases before SARS-CoV-2 infection. In a series of case studies, the authors observed that older adults with a history of neuropsychiatric disease had delirium as a severe manifestation of COVID-19. Subsequently, these individuals manifested motor and cognitive changes, such as agitation, rigidity, abulia, and alogia. Parallel to neurological and cognitive changes, patients showed an increase in systemic C-reactive protein (CRP). The authors suggest that these patients should be monitored in order to promote mental health and diagnose forehand the development or increase neuropsychiatric and cognitive damages [58].

\section{Can Disorders Increase the Severity of COVID-19 Symptoms?}

Before the pandemic, some populations already lived with the effects of psychiatric disorders on their daily lives, directly impacting their quality of life. Severe depressive conditions are estimated to reduce life expectative from 13 to 30 years due to inflammatory and neurological complications associated with the disease $[8,59]$. Neuroinflammation is a biological factor present in the pathology of the MDD [60] and a relevant aggravating factor of the COVID-19 [21]. IL- $1 \beta$ is one of the critical pro-inflammatory mediators in trauma, abuse, childhood maltreatment, and anxiety disorder. Besides, IL- $1 \beta$ expression tends to change according to the severity of anxiety and depressive symptoms [61]. IL-1 $\beta$ is also associated as a pathway of inflammation induced by caspases' activation during COVID-19 infection [62]. According to clinical findings, individuals who already have pathologies that increase inflammatory cytokines expression, including IL-1 $\beta$, may experience worsening of COVID-19 inflammatory symptoms [62].

An important mediator of inflammatory cytokines and the immune system is the HPA axis, often deregulated in individuals with stress-related psychiatric disorders [51, 63]. Studies with rodents show that corticosterone levels are positively related to vulnerability to stress $[64,65]$. MDD is one of the pathologies associated with chronic stress, and, therefore, in many patients, the HPA axis is unregulated, also involving the dysregulation of the inflammatory process. In addition to activating the inflammatory pathways, cytokines act as regulators of the HPA axis $[60$, 66] and can induce glucocorticoid resistance [60]. This condition harms the negative feedback of the axis, which triggers a vicious cycle, leading to a chronic process involved in inflammation and organic dysfunctions [56].

Prolonged stress situations cause an overload of corticosteroids [60] and increase the susceptibility to contracting viral infections. Long-lasting changes in the HPA axis and the immune system can trigger epigenetic changes in the innate immune system's stem cells and adaptive immunity from a viral infection. This situation can culminate in prolonged neuropsychiatric changes after the disease. On the other hand, the effects of prolonged stress on the HPA axis can induce epigenetic changes that make the individual more susceptible to the worsening of viral infections and greater susceptibility to activation of latent viruses [56, 67]. Immunosenescence naturally causes dysregulation in the immune system, and, when associated with stress, this vulnerability is amplified [68]. The significant decrease in circulating B cells and antibodies [69] as well as an increase in IL-6 and CRP [70] are factors that contribute to impaired immune responses in the elderly $[68,71,72]$. These conditions increase the risk of mortality associated with infections [68]. IL-6 and CRP levels systematically increase in an age-dependent manner [70]. CRP is also high in older people with depression and dementia [73]. The biological mechanisms involved in depression and anxiety disorders, associated with advanced age, become important aggravating factors for the progression of COVID-19.

CRH elevates circulating pro-inflammatory cytokines through monocytic activation, beyond its function on the HPA axis. Animals subjected to stress express high levels of circulating IL-1 $\beta$, IL-17, and TNF- $\alpha$ [63]. Individuals who contracted COVID-19 have a higher rate of platelet-derived growth factor (PDGF), TNF- $\alpha$, and vascular endothelial growth factor (VEGF), when compared with uninfected people [21]. These events suggest a greater severity and fatality of COVID19 in people who have comorbidities induced by stressful events, mainly dementia and psychiatric disorders [74].

Renin-angiotensin-aldosterone system (RAAS) also is a mechanism involved in COVID-19 infection. This system is responsible for regulating the variation of extracellular fluid and blood pressure. Researches indicate that RAAS is directly 
affected by SARS-CoV-2 because the angiotensin-converting enzyme 2 (ACE2), a regulator of the vasopressor axis, is the molecule that allows the communication, entry, and replication of the virus into the cell $[56,75]$.

ACE2 is expressed in all districts of the human body, including brain tissue, comprising a possible explanation for the central nervous system (CNS) involvement, although studies are still speculative. It is suggested that therapies that involve a decrease in the expression of ACE2 may lead to hyperactivation of the renin-angiotensin system and the HPA axis and, in the long term, culminate in MDD, anxiety, and post-traumatic stress [56]. Studies with mice revealed that overexpression of ACE2 in CRH target cells decreased anxiety in plus-maze and open field tests [76].

Some populations may be more prone to develop neurological problems as a result of COVID-19. Brain aging is the leading risk factor for neurodegenerative diseases and cognitive decline. Such clinical conditions increase the risk of complications associated with COVID-19. Felice et al. [77] observed different types of coronavirus (CoV-OC43 and $\mathrm{CoV}$ $229 \mathrm{E}$ ) in brain tissue and cerebrospinal fluid in patients with Parkinson's disease (PD). PD mainly affects the elderly and induces a decrease in movement. Thus, repercussions of their pathophysiology can compromise the respiratory system, worsening the infectious condition of Covid-19 [78].

Hypertension and diabetes are other documented aggravations regarding COVID-19 [18]. Among the mechanisms that associate the severity of Covid-19 with diabetes is the impaired immune response and pancreatic damage caused by SARSCoV-2 [79]. Individuals with mental disorders often have changes in the glycemic index [30]. Some mental disorders such as depression and anxiety contribute to the development and progression of metabolic and cardiovascular disorders [56]. Also, individuals with dementia have high risk of developing cardiovascular disease, diabetes, and pneumonia [53].

\section{Can COVID-19 Worsen the Symptoms of Psychiatric Disorders in the Elderly?}

Considering the unfavorable consequences of a pandemic, the first signs of psychic shock appear. Excess of information can intensify emotional instability and lead to paranoia [3]. The situation worsens when someone relative is affected, triggering periods of extreme sadness and depression [4]. The apprehension about their vulnerability and the maintenance of security contribute to panic episodes. Those who already have a mental disorder are more conducive to suffering from stress and may be more susceptible to relapse or worsening of preexisting mental health situations [80].

The remodeling of relationships puts at risk the elderly who are already prone to depression and anxiety and increase the possibilities of "amplifying pre-existing conditions" [81].
Mehra et al. [5] report the case of an older adult who has been with depression for more than 50 years. For 3 weeks, he was too anxious about COVID-19. On physical examination, no abnormalities were identified. The mental status exam pointed to "excessive concern about becoming infected with COVID19." It was necessary to increase the dose of antidepressant and psychotherapy sessions to control the manifestations. The same author brings the case of an older woman diagnosed with recurrent depression for 7 years, without medication and symptom control. After the outbreak of COVID-19, she showed anxiety about her health. The patient needed emergency care due to depressive and psychotic symptoms and treatment with antidepressants and antipsychotics.

Both cases exemplify that the pandemic's social changes cause emotional suffering and frustration in older people with mental disorders [29]. Simple day-to-day activities become stressors, and, with the increasing burden of health systems, there is an imminent risk of discontinuing the monitoring of elderly in mental suffering.

Kavoor [30] points to recurrences that result in noncompliance of preventive actions, such as social detachment, lack of personal hygiene, and interruption of adequate treatment without intentions of seeking specialized help. Even for those who do not have a psychiatric condition, isolation is a resilience challenge. It is estimated that between one-third and half of the population will develop psychopathological manifestations in this period if preventive measures are not taken to minimize the impact [82].

In addition to the emotional burden caused by isolation, the reduction or complete interruption of physical activity also comes up with an additional charge harmful to health. Thus, the older adult isolated in an impoverished environment of regular social, cognitive, and sensorimotor stimulation will worsen health conditions and, even, can lead to a lonely and premature death [8].

On the other hand, it is crucial to think about older people who have suffered contamination from SARS-CoV-2. There is a hypothesis that this virus can leave sequels for those who suffered infection and possibly increase the vulnerability to develop and exacerbate mental disorders [83]. The SARS$\mathrm{CoV}-2$ infection can lead to a typical cascade of responses to stress, such as activation of the HPA axis and other processes related to homeostasis maintenance [56]. With the activation of the HPA axis, the hypothalamic hormone (CRH) stimulates the release by the pituitary of adrenocorticotropic hormone (ACTH), which stimulates the release of corticosteroids by the adrenal glands. During a normal response, corticosteroids reach their brain receptors and trigger a negative feedback signal to interrupt the release of hypothalamic and pituitary hormones, reducing the adrenals' stimulation and, consequently, the release of corticosteroids [84]. However, suppose the activation of the HPA axis is exacerbated and prolonged. In that case, the negative feedback loop can be impaired, 
leading to an excessive increase in corticosteroids' levels with several physiological changes [56].

Exacerbated and chronic activation of the HPA axis and the immune system are responsible for several psychiatric disorders, such as anxiety, depression, and post-traumatic stress disorder, among others $[56,60]$. In addition to the activation of the endocrine and immune systems, studies on SARS disease have observed that the virus installs in several organs, including the pituitary, directly affecting the HPA axis [85].

As already reported, COVID-19 also triggers a potent immune response at the systemic level. At the forefront of immune activation are pro-inflammatory cytokines, which signal the recruitment of macrophages. With chemotaxis, monocytes and neutrophils reach the signaled infectious site [84]. Deaths from sepsis are often associated with acute respiratory distress syndrome (ARDS) and the diagnosis of SARS-CoV-2 [86]. As the infection progresses, the blood-brain barrier may disrupt, causing damage to the CNS. Neuroinfection, psychiatric disorders, and degenerative diseases are some of the body's responses to the virus. When underlying conditions already exist, these can be aggravated [77, 87].

In addition to the peripheral cytokines storm and activation of the HPA axis, the SARS-CoV-2 virus, like other SARS coronaviruses, appears to access the CNS, which may trigger an infectious and inflammatory process directly in regions of the nervous system. Although evidence is still lacking, several neurological manifestations suggest that the SARS-CoV-2 accesses and causes changes in the CNS and may be responsible for direct interference in the brain stem's respiratory regulation areas [88]. Studies on COVID-19 are still preliminary about tissues and organs directly affected by the virus. However, an in vitro study noted that the virus could infect neural cells. The authors argue that this possibility may be related to the nervous system's symptoms and developed by several critically ill patients [89]. In addition to the degree of similarity with other coronaviruses that access the CNS, anosmia, and dysgeusia present in many infected individuals, even before respiratory damage, are symptoms that are possibly related to the entry of SARS-CoV-2 by the olfactory nerve [90]. More recent studies suggest that impairments in the senses of taste and smell are related to the disruption of sensory neural mechanisms, affecting the chemosynthesis of infected individuals [91]. Therefore, SARS-CoV-2 may activate neuroinflammatory processes in the nervous system regions, inducing behavioral changes or other neurological damage. These effects of COVID-19 may also exacerbate other psychiatric disorders already in place before of infection.

Considering that the main physiological mechanisms stimulated by SARS-CoV-2 are familiar to those elicited by chronic stress, psychiatric disorders, and advancing age, COVID-19 can intensify, both by triggering limbic brain mechanisms in the combat of stress and by triggering physiological mechanisms inherent to disease and age situations.

\section{Procedures and Health Care to Reduce the Damage to Mental Health of the Elderly Through Pandemic}

Among the treatments to improve quality of life, social interaction and therapy groups are essential strategies for coping with the symptoms of dementia and mental disorders. The social connection is directly related to neural and hormonal mechanisms. The feeling of loneliness caused by isolation results in a lowering of mood and cognition, altering the regulation of inflammatory responses in the body, which damages the immune system, the ability to concentrate, and sleep habits [31].

Given the context of worsening psychiatric symptoms driven by the pandemic, it is crucial to focus on the necessary care to maintain the mental health of the elderly, considering the characteristics of people who already lived with suffering, as well as those who developed emotional damage. Among the most vulnerable groups are those who live in nursing homes or recovery clinics. Because their assistance requires several professionals, they are unable to perform isolation [4]. In general, measures to prevent the risks of mental illness must offer the necessary support thanks to family and social support networks, in addition to the care provided by health professionals and services. In this sense, it is imperative to convey information to prevent psychiatric effects, considering that this information can be understandable and straightforward to reduce barriers and facilitate understanding.

The elderly and their family members must manage their daily needs, such as the continuous use of medications and guidance from health professionals regarding pharmacological and non-pharmacological treatments. It is beneficial in this period that the elderly can perform physical activity at home and that they seek ways to communicate with family and friends during this period. It is convenient for those who have technological tools to use them to maintain safe distance contact [92].

Cellular devices and computers can compose crucial mental health care tools during this period. Its use facilitates communication with the family and social circle, counseling sessions, and information on medical and psychological needs [93]. Direct contact, based on calls to mental health centers, can screen the symptoms of anxiety and depression and reduce the impact of pandemic context in the mental health of the elderly [94].

It is well suited to maintain the daily activities performed by the elderly. It is also essential to create other practices that enrich their daily lives, create leisure moments at home, and use painting, music, literature, and other options that they have easier access. These recommendations provide an opportunity for the elderly to remain active and healthy when contemplating the biopsychosocial dimensions. Such actions must be integrated and carried out as long as they are adequately supported by health professionals and public policies [95]. 
On the other hand, it should be in mind that not all older people can ease the pandemic moment's tension. For some, even exercise is challenging due to confinement [4]. There are several situations of social and individual vulnerability. The low capacity for resilience can impair mental health. Looking for the different demands is necessary to integrate the health services that provide care at that time and palliative actions and to insert mental health at the epicenter of the care provided [3].

It is also essential to observe simple guidelines that can help maintain mental health in the elderly, reduce stress and anxiety, and promote self-care for the elderly and those around them during the COVID-19 period. Among the guidelines is to remain physically healthy, including physical exercises, among other attitudes [96].

Physical exercises can be a strategy for controlling MDD and other psychiatric disorders and other chronic diseases, which, besides being associated with the conditions, can predispose greater severity to the symptoms of COVID-19. With the regular practice of physical activities, many physiological mechanisms involved in the endocrine axis and immune function can be improved, reducing both the severity of psychiatric disorders and the possible severity of SARS-CoV-2 infection. Among the critical mechanisms affected by regular physical exercise are the reduction of oxidative stress and pro- inflammatory cytokines, with consequent beneficial changes in the biological processes of degradation of tryptophan. The increased availability of tryptophan and, consequently, serotonin levels are important factors for reducing symptoms related to depression and other disorders. Furthermore, the reduction of peripheral inflammation and neuroinflammation improves brain dynamics in the limbic circuit and may be achieved through regular physical exercises [60].

Here, in addition to not intending to exhaust the possibilities of improving the quality of life, it is also important to highlight that any initiative and care is impossible for a large percentage of the elderly population. Therefore, these are issues that will still demand public health concerns and future research needs.

\section{Considerations and Conclusions}

Researches conducted during the initial phase of COVID-19 indicate that the impact on mental health was already moderate to severe, and many people had manifestations of anxiety and depression [97]. During pandemics, it is common for government efforts to be directed towards physical signs. However, it is also important that both health institutions

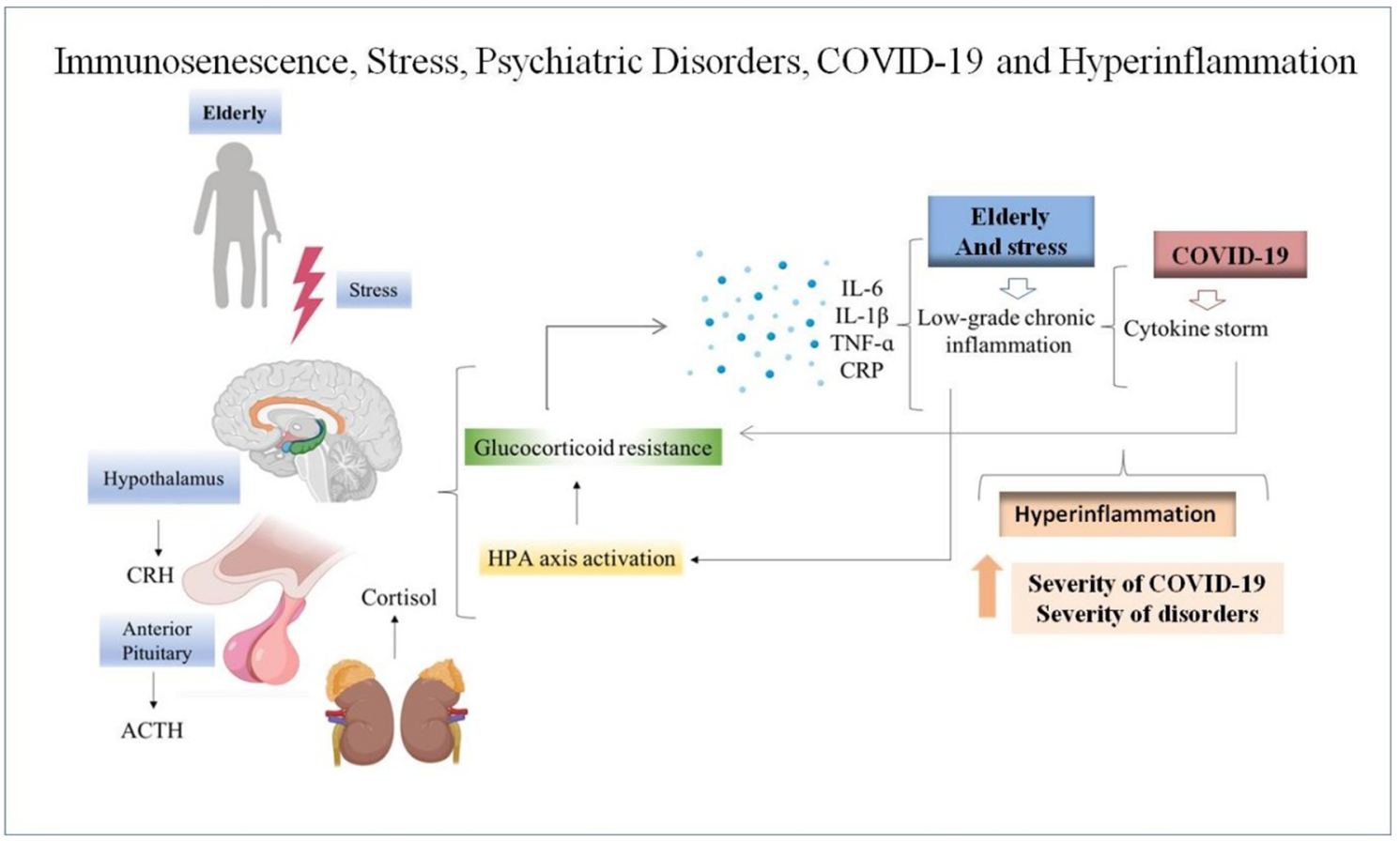

Fig. 2 Immunosenescence in aging is accompanied by a chronic inflammatory state. Simultaneously, chronic stress during a pandemic may deregulate negative feedback from the hypothalamic-pituitaryadrenal (HPA) axis. The chronic increase in glucocorticoids (cortisol) is accompanied by an increase in cytokines and other pro-inflammatory molecules. In turn, pro-inflammatory cytokines contribute to the imbalance of the HPA axis, which can culminate in resistance to glucocorticoids, contributing to an increase or maintenance of a chronic proinflammatory state. These associated conditions predispose the elderly to trigger or worsen major depressive disorder (MDD) and other psychiatric disorders, as well as make them more vulnerable to the severity of COVID-19 symptoms. On the other hand, the pro-inflammatory cytokine storm, a condition of the severity of SARS-CoV-2 infection, can trigger or worsen psychiatric disorders in the elderly. CRH $\rightarrow$ corticotropinreleasing hormone; ACTH $\rightarrow$ adrenocorticotropic hormone; IL $\rightarrow$ interleukin; TNF- $\alpha \rightarrow$ tumor-necrosis factor- $\alpha$; CRP $\rightarrow$ C-reactive protein. Images were extracted from Biorender app 
and science are aware of the consequences related to the mental health of the elderly [81]. Although social isolation is the most critical measure in coping with SARS-CoV-2, attention to mental health is extremely necessary because suicide risks are also high [92].

Several studies on the biological processes that emerge with age, stress, psychiatric disorders, and the SARS-CoV-2 infection suggest that there may be a synergism in these situations, leading to an increase in severity, both for COVID-19 and for damage mental health of the elderly. The stress involved in the psychosocial environment and the condition inherent to the disease triggers the HPA axis and the immune system's inflammatory processes. Advanced age can bring immunosenescence, which is also related to a chronic inflammatory degree. These associated conditions alone can culminate in a higher degree of chronic inflammation, predisposing the elderly to psychiatric disorders, such as anxiety and MDD, among others. Studies report that social isolation has been linked to increased depression and suicide, increased inflammation, and reduced immune response to viral infections. These conditions also predispose the elderly to more severe conditions when they contract SARS-CoV-2 [94].

It is essential to pay attention to the mental damage resulting from the synergism between a possible unfavorable biological condition related to aging and a SARS-CoV-2 infection. This combination can converge to a state of hyper inflammation, a more significant change in the function of the HPA axis, and the onset or worsening of psychiatric disorders. (Fig. 2).

For the inadequate care provided to the mentally vulnerable population during the pandemic, health policymakers' collaboration is necessary to create and implement quality and accessible services during the crisis, especially for the elderly [87], a group especially fragile about their mental state. For maintenance, some actions can be implemented based on the Active Aging Policy, adopted by several countries, which focuses on health promotion and protection [98]. Each country must have public policies geared to its needs to attend different specificities. In addition to social measures, there is an urgent need to strengthen geriatric technical and professional training, due to the imminent risk of worsening psychopathological and neurodegenerative conditions [81].

In addition to the suggestions for attention and care for the elderly during the pandemic, it is essential to note that both health organizations worldwide and science should consider an increase in mental impairment in the elderly population worldwide from the conditions listed in this and other studies. Paying attention to these issues is essential for studies and ways to understand and alleviate mental suffering and further damage to the elderly's health.

Acknowledgments The Translational Psychiatry Program (USA) is funded by the Department of Psychiatry and Behavioral Sciences,
McGovern Medical School, The University of Texas Health Science Center at Houston (UTHealth). Translational Psychiatry Laboratory (Brazil) is one of the members of the Center of Excellence in Applied Neurosciences of Santa Catarina (NENASC). Its research is supported by grants from CNPq (ZMI, JQ and GZR), FAPESC (ZMI, JQ, and GZR), UFFS (ZMI), Instituto Cérebro e Mente (JQ and GZR), and UNESC (JQ and GZR). JQ is a $1 \mathrm{~A} \mathrm{CNPq} \mathrm{Research} \mathrm{Fellow.}$

Authors' Contributions All authors participated in the construction of the review. REG, MEDM, and AGB searched for references and started writing the manuscript; ARM wrote the last topic and contributed others; ZMI outlined the article and wrote the part of COVID-19's physiological mechanisms; GZR helped in writing about stress and inflammation; JQ reviewed and made suggestions for improving the manuscript; JQ, GZR, and ZMI reviewed the final manuscript. All authors read and approved the manuscript.

Data Availability Not applicable in this study.

Compliance with Ethical Standards This study complies with ethical standards.

Conflict of Interest The authors declare that they have no conflict of interest.

Consent to Participate Not applicable in this study.

Consent for Publication Not applicable in this study.

\section{References}

1. Wu Z, McGoogan JM (2020) Characteristics of and important lessons from the coronavirus disease 2019 (COVID-19) outbreak in China: summary of a report of 72314 cases from the Chinese Center for Disease Control and Prevention. JAMA 323:12391242. https://doi.org/10.1001/jama.2020.2648

2. Shahid Z, Kalayanamitra R, McClafferty B (2020) COVID-19 and older adults: what we know. J Am Geriatr Soc 68:926-929. https:// doi.org/10.1111/jgs. 16472

3. Banerjee D (2020) The COVID-19 outbreak: Crucial role the psychiatrists can play. Asian J Psychiatr 50:102014. https://doi.org/10. 1016/j.ajp.2020.102014

4. Brown EE, Kumar S, Rajji TK, Pollock BG, Mulsant BH (2020a) Anticipating and mitigating the impact of the COVID-19 pandemic on Alzheimer's disease and related dementias. Am J Geriatr Psychiatry 28:712-721. https://doi.org/10.1016/j.jagp.2020.04.010

5. Mehra A, Rani S, Sahoo S, Parveen S, Singh AP, Chakrabarti S, Grover S (2020) A crisis for elderly with mental disorders: relapse of symptoms due to heightened anxiety due to COVID-19. Asian J Psychiatr 51:10211. 10.1016\%2Fj.ajp.2020.102114

6. World Health Organization (2017) Mental health of older adults. https://www.who.int/en/news-room/fact-sheets/detail/mentalhealth-of-older-adults.

7. Cipriani G, Fiorino MD (2020) Access to care for dementia patients suffering from COVID-19. Am J Geriatr Psychiatry 28:796-797. https://doi.org/10.1016/j.jagp.2020.04.009

8. Plagg B, Engl A, Piccoliori G, Eisendle K (2020) Prolonged social isolation of the elderly during COVID-19: between benefit and damage. Arch Gerontol Geriatr 89:104086. 10.1016\%2Fj.archger.2020.104086 
9. Hao Y, Jian-Hua C, Yi-Feng X (2020) Patients with mental health disorders in the COVID-19 epidemic. Lancet Psychiat 7:21. https:// doi.org/10.1016/S2215-0366(20)30090-0

10. Serafini G, Bondi E, Locatelli C, Amore M (2020) Aged patients with mental disorders in the COVID-19 era: the experience of northern Italy. Am J Geriatr Psychiatry 28:794-795. https://doi. org/10.1016/j.jagp.2020.04.015

11. World Health Organization (2020a) World Health Organization: coronavirus disease 2019 (COVID-19) situation report - 194, 2020. https://www.who.int/emergencies/diseases/novelcoronavirus-2019/situation-reports. Accessed 11 Dec 2020

12. Menachery VD, Yount BL Jr, Debbink K, Agnihothram S, Gralinski LE, Plante JA, Graham RL, Scobey T et al (2015) A SARS-like cluster of circulating bat coronaviruses shows potential for human emergence. Nat Med 21:1508-1513. https://doi.org/10. 1038/nm.3985

13. Zhou P, Yang XL, Wang XG, Hu B, Zhang L, Zhang W, Si HR, Zhu $Y$ et al (2020a) A pneumonia outbreak associated with a new coronavirus of probable bat origin. Nature 579:2070-2273. https:// doi.org/10.1038/s41586-020-2012-7

14. Moccia F, Gerbino A, Lionetti V, Miragoli M, Munaron LM, Pagliaro P, Pasqua T, Penna C et al (2020) COVID-19-associated cardiovascular morbidity in older adults: a position paper from the Italian Society of Cardiovascular Researches. GeroScience 20:129. https://doi.org/10.1007/s11357-020-00198-w

15. Chen IY, Moriyama M, Chang MF, Ichinohe T (2019) Severe acute respiratory syndrome coronavirus viroporin 3a activates the NLRP3 inflammasome. Front Microbiol 10:50. 10.3389\%2Ffmicb.2019.00050

16. Wang L, He W, Yu X, Hu D, Bao M, Liu H, Zhou J, Jiang H (2020a) Coronavirus disease 2019 in elderly patients: characteristics and prognostic factors based on 4-week follow-up. J Infect 80: 639-645. https://doi.org/10.1016/j.jinf.2020.03.019

17. Statista Research Department (2020) Coronavirus (COVID-19) death rate in Italy as of April 9, 2020, by age group. https://www. statista.com/statistics/1106372/coronavirus-death-rate-by-agegroup-italy/.

18. Zhou F, Yu T, Du R, Fan G, Liu Y, Liu Z, Xiang J, Wang Y et al (2020b) Clinical course and risk factors for mortality of adult inpatients with COVID-19 in Wuhan, China: a retrospective cohort study. Lancet 395:1054-1062. https://doi.org/10.1016/S01406736(20)30566-3

19. Tay MZ, Poh CM, Rénia L, MacAry PA, Ng LFP (2020) The trinity of COVID-19: immunity, inflammation and intervention. Nat Rev Immunol 20:1-12. https://doi.org/10.1038/s41577-020-0311-8

20. Yang M (2020) Cell pyroptosis, a potential pathogenic mechanism of 2019-nCoV infection. SSRN Electron J. https://doi.org/10.2139/ ssrn. 3527420

21. Huang C, Wang Y, Li X, Ren L, Zhao J, Hu Y, Zhang L, Fan G et al (2020) Clinical features of patients infected with 2019 novel coronavirus in Wuhan, China. Lancet 395:497-506. https://doi.org/10. 1016/S0140-6736(20)30183-5

22. Gorse GJ, Patel GB, Vitale JN, O'Connor TZ (2010) Prevalence of antibodies to four human coronaviruses is lower in nasal secretions than in serum. Clin Vaccine Immunol 17:1875-1880. https://doi. org/10.1128/CVI.00278-10

23. Zheng Y, Li R, Liu S (2020) Immunoregulation with mTOR inhibitors to prevent COVID-19 severity: a novel intervention strategy beyond vaccines and specific antiviral medicines. J Med Virol 92: 1495-1500. https://doi.org/10.1002/jmv.26009

24. Diao B, Wang C, Tan Y, Chen X, Liu Y, Ning L, Chen L, Li M et al (2020) Reduction and functional exhaustion of T cells in patients with coronavirus disease 2019 (COVID-19). Front Immunol 11: 827. https://doi.org/10.3389/fimmu.2020.00827

25. Bektas A, Schurman SH, Sen R, Ferrucci L (2017) Human T cell immunosenescence and inflammation in aging. J Leukoc Biol 102: 977-988. https://doi.org/10.1189/jlb.3ri0716-335r
26. Neurath MF (2020) Covid-19 and immunomodulation in IBD. Gut 69:1335-1342. https://doi.org/10.1136/gutjnl-2020-321269

27. Ostir GV, Goodwin JS (2006) High anxiety is associated with an increased risk of death in an older tri-ethnic population. J Clin Epidemiol 59:534-540. https://doi.org/10.1016/j.jclinepi.2005.10. 008

28. Sun K, Chen J, Viboud C (2020) Early epidemiological analysis of the coronavirus disease 2019 outbreak based on crowdsourced data: a population-level observational study. The Lancet Digit Health 2: 201-208. https://doi.org/10.1016/S2589-7500(20)30026-1

29. Xiang YT, Yang Y, Li W, Zhang L, Zhang Q, Cheung T, Ng CH (2020) Timely mental health care for the 2019 novel coronavirus outbreak is urgently needed. Lancet Psychiat 7:228-229. https:// doi.org/10.1016/S2215-0366(20)30046-8

30. Kavoor AR (2020) COVID-19 in people with mental illness: challenges and vulnerabilities. Asian J Psychiatr 51:102051. 10.1016\%2Fj.ajp.2020.102051

31. Santini ZI, Jose PE, Cornwell EY, Koyanagi A, Nielsen L, Hinrichsen C, Meilstrup C, Madsen KR et al (2020) Social disconnectedness, perceived isolation, and symptoms of depression and anxiety among older Americans (NSHAP): a longitudinal mediation analysis. Lancet Public Health 5:62-70. https://doi.org/10. 1016/S2468-2667(19)30230-0

32. Alexopoulos GS (2019) Mechanisms and treatment of late-life depression. Transl Psychiatry 9:188. 10.1038\%2Fs41398-019-05146

33. Abelaira HM, Reus GZ, Neotti MV, Quevedo J (2014) The role of mTOR in depression and antidepressant responses. Life Sci 101: 10-14. https://doi.org/10.1016/j.lfs.2014.02.014

34. Bauer K, Schwarzkopf L, Graessel E, Holle R (2014) A claims data-based comparison of comorbidity in individuals with and without dementia. BMC Geriatr 14:1-13. https://doi.org/10.1186/14712318-14-10

35. Miller AH, Raison CL (2016) The role of inflammation in depression: from evolutionary imperative to modern treatment target. Nat Rev Immunol 16:22-34. https://doi.org/10.1038/nri.2015.5

36. Song C, Wang H (2011) Cytokines mediated inflammation and decreased neurogenesis in animal models of depression. Prog Neuro-Psychopharmacol Biol Psychiatry 35:760-768. https://doi. org/10.1016/j.pnpbp.2010.06.020

37. Dilger RN, Johnson RW (2008) Aging, microglial cell priming, and the discordant central inflammatory response to signals from the peripheral immune system. J Leukoc Biol 84:932-939. https://doi. org/10.1189/jlb.0208108

38. Lucin KM, Wyss-Coray T (2009) Immune activation in brain aging and neurodegeneration: too much or too little? Neuron 64:110-122. https://doi.org/10.1016/j.neuron.2009.08.039

39. Feltes KP, Doorduin J, Klein HC, Juárez-Orozco LE, Diercky RAJO, Moriguchi-Jeckel CM, Vries EFJ (2017) Antiinflammatory treatment for major depressive disorder: implications for patients with an elevated immune profile and non-responders to standard antidepressant therapy. J Psychopharmacol 31(9):1149 1165. https://doi.org/10.1177/0269881117711708

40. Miller AH, Maletic V, Raison CL (2009) Inflammation and its discontents: the role of cytokines in the pathophysiology of major depression. Biol Psychiatry 65:732-741. https://doi.org/10.1016/j. biopsych.2008.11.029

41. Ignácio ZM, Réus GZ, Arent CO, Abelaira HM, Pitcher MR, Quevedo J (2016) New perspectives on the involvement of mTOR in depression as well as in the action of antidepressant drugs. Br J Clin Pharmacol 82:1280-1290. https://doi.org/10. $1111 /$ bcp. 12845

42. Nowak W, Grendas LN, Sanmarco LM, Estecho IG, Arena ÁR, Eberhardt N, Rodante DE, Aoki MP et al (2019) Pro-inflammatory monocyte profile in patients with major depressive disorder and suicide behaviour and how ketamine induces anti-inflammatory 
M2 macrophages by NMDAR and mTOR. EBioMedicine 50:290305. https://doi.org/10.1016/j.ebiom.2019.10.063

43. Abelaira HM, Réus GZ, Ignácio ZM, Dos Santos MAB, de Moura AB, Matos D, Demo JP, da Silva JBI et al (2017) Ketamine exhibits different neuroanatomical profile after mammalian target of rapamycin inhibition in the prefrontal cortex: the role of inflammation and oxidative stress. Mol Neurobiol 54:5335-5346. https://doi. org/10.1007/s12035-016-0071-4

44. Zegeye MM, Lindkvist M, Fälker K, Kumawat AK, Paramel G, Grenegård M, Sirsjö A, Ljungberg LU (2018) Activation of the JAK/STAT3 and PI3K/AKT pathways are crucial for IL-6 transsignaling-mediated pro-inflammatory response in human vascular endothelial cells. Cell Commun Signal 16:55. https://doi.org/10. 1186/s12964-018-0268-4

45. Ramaiah MJ (2020) mTOR inhibition and p53 activation, microRNAs: the possible therapy against pandemic COVID-19. Gene Rep 20:100765. https://doi.org/10.1016/j.genrep.2020. 100765

46. Strawbridge R, Hodsoll J, Powell TR, Hotopf M, Hatcha SL, Breen G, Cleare AL (2019) Inflammatory profiles of severe treatmentresistant depression. J Affect Disord 246:42-51. https://doi.org/ 10.1016/j.jad.2018.12.037

47. Strawbridge R, Young AH, Cleare AJ (2017) Biomarkers for depression: recent insights, current challenges and future prospects. Neuropsychiatr Dis Treat 13:1245-1262. https://doi.org/10.2147/ ndt.s 114542

48. Frank MG, Weber MD, Watkins LR, Maier SF (2015) Stress sounds the alarmin: the role of the danger-associated molecular pattern HMGB1 in stress-induced neuroinflammatory priming. Brain Behav Immun 48:1-7. https://doi.org/10.1016/j.bbi.2015. 03.010

49. Prinz M, Priller J (2014) Microglia and brain macrophages in the molecular age: from origin to neuropsychiatric disease. Nat Rev Neurosci 15:300-312. https://doi.org/10.1038/nrn3722

50. Yirmiya R, Rimmerman N, Reshef R (2015) Depression as a microglial disease. Trends Neurosci 38:637-658. https://doi.org/ 10.1016/j.tins.2015.08.001

51. Slavich GM, Irwin MR (2014) From stress to inflammation and major depressive disorder: a social signal transduction theory of depression. Psychol Bull 140:774-815 https://pubmed.ncbi.nlm. nih.gov/24417575/

52. Friedler B, Crapser J, McCullough L (2015) One is the deadliest number: the detrimental effects of social isolation on cerebrovascular diseases and cognition. Acta Neuropathol 129:493-509. https:// doi.org/10.1007/s00401-014-1377-9

53. Brown E, Gray R, Monaco SL, O'Donoghueab B, Nelson B, Thompso A, Franceya S, McGorry P (2020b) The potential impact of COVID-19 on psychosis: a rapid review of contemporary epidemic and pandemic research. Schizophr Res 222:79-87. https:// doi.org/10.1016/j.schres.2020.05.005

54. Colizzi M, Bortoletto R, Silvestri M, Mondini F, Puttini E, Cainelli C, Gaudino R, Ruggeria M et al (2020) Medically unexplained symptoms in the times of Covid-19 pandemic: a case-report. Brain Behav Immun Health 5:1000734. https://doi.org/10.1016/j. bbih.2020.100073

55. Dennison U, McKernan D, Cryan J, Dinan T (2012) Schizophrenia patients with a history of childhood trauma have a proinflammatory phenotype. Psychol Med 42:1865-1871. https://doi. org/10.1017/s0033291712000074

56. Steenblock C, Todorov V, Kanczkowski W, Eisenhofer G, Schedl A, Wong ML, Licinio J, Bauer M et al (2020) Severe acute respiratory syndrome coronavirus 2 (SARS-CoV-2) and the neuroendocrine stress axis. Mol Psychiatry 25:1611-1617. https://doi.org/10. 1038/s41380-020-0758-9

57. Tylee DS, Chandler SD, Nievergelt CM, Liu X, Pazol J, Woelk CH, Lohr JB, Kremen WS et al (2015) Blood-based gene-expression biomarkers of post-traumatic stress disorder among deployed marines: a pilot study. Psychoneuroendocrinology 51:472-494. https://doi.org/10.1016/j.psyneuen.2014.09.024

58. Beach SR, Praschan NC, Hogan C, Dotson S, Merideth F, Kontos N, Fricchione GL, Smith FA (2020) Delirium in COVID-19: a case series and exploration of potential mechanisms for central nervous system involvement. Gen Hosp Psychiatry 65:47-53. https://doi. org/10.1016/j.genhosppsych.2020.05.008

59. Schneider F, Erhart M, Hewer W, Loeffler LA, Jacobi F (2019) Mortality and medical comorbidity in the severely mentally ill-a German registry study. Dtsch Arztebl Int 116:405-441. https://doi. org/10.3238/arztebl.2019.0405

60. Ignácio ZM, Da Silva RS, Plissari ME, Quevedo J, Réus GZ (2019) Physical exercise and Neuroinflammation in major depressive disorder. Mol Neurobiol 56:8323-8335. https://doi.org/10.1007/ s12035-019-01670-1

61. Grasser LR, Burghardt P, Daugherty AM, Amirsadri A, Javanbakht A (2020) Inflammation and trauma-related psychopathology in Syrian and Iraqi refugees.Behav Sci (Basel) 10(4):75. https://doi. org/10.3390/bs 10040075

62. Parisi V, Leosco D (2020) Precision medicine in COVID-19: IL-1 $\beta$ a potential target. JACC Basic Transl Sci 5:543-544. https://doi. org/10.1016/j.jacbts.2020.04.006

63. Lu Y, Ho CS, Liu X, Chua AN, Wang W, McIntyre RS, Ho RC (2017) Chronic administration of fluoxetine and pro-inflammatory cytokine change in a rat model of depression. PLoS One 12:1-14. https://doi.org/10.1371/journal.pone.0186700

64. Gómez-Lázaro E, Arregi A, Beitia G, Vegas O, Azpiroz A, Garmendia L (2011) Individual differences in chronically defeated male mice: behavioral, endocrine, immune, and neurotrophic changes as markers of vulnerability to the effects of stress. Stress 14:537-548. https://doi.org/10.3109/10253890.2011.562939

65. Pérez-Tejada J, Arregi A, Gómez-Lázaro E, Vegas O, Azpiroz A, Garmendia L (2013) Coping with chronic social stress in mice: hypothalamic-pituitary-adrenal/ sympathetic-adrenal-medullary axis activity, behavioral changes and effects of antalarmin treatment: implications for the study of stress-related psychopathologies. Neuroendocrinology 98:73-88. https://doi.org/10.1159/000353620

66. Hodes GE, Kana V, Menard C, Merad M, Russo SJ (2015) Neuroimmune mechanisms of depression. Nat Neurosci 18:13861393. https://doi.org/10.1038/nn.4113

67. Yan C, Luo Z, Li W, Li X, Dallmann R, Kurihara H, Li Y, He RR (2020) Disturbed Yin-Yang balance: stress increases the susceptibility to primary and recurrent infections of herpes simplex virus type 1. Acta Pharm Sin B 10:383-398. https://doi.org/10.1016/j. apsb.2019.06.005

68. Smetana J, Chlibek R, Shaw J, Splino M, Prymula R (2018) Influenza vaccination in the elderly. Hum Vaccin Immunother 14: 540-549. https://doi.org/10.1080/21645515.2017.1343226

69. Pinti M, Appay V, Campisi J, Frasca D, Fülöp T, Sauce D, Larbi A, Weinberger B et al (2016) Aging of the immune system: focus on inflammation and vaccination. Eur J Immunol 46:2286-2301. https://doi.org/10.1002/eji.201546178

70. Puzianowska-Kuźnicka M, Owczarz M, Wieczorowska-Tobis K, Nadrowski P, Chudek J, Slusarczyk P, Skalska A, Jonas M, Franek E, Mossakowska M (2016) Interleukin-6 and C-reactive protein, successful aging, and mortality: the PolSenior study. Immun Ageing 13:21. 10.1186\%2Fs 12979-016-0076-x

71. Gravenstein S, Drinka P, Duthie EH, Miller BA, Brown CS, Hensley M, Circo R, Langer E et al (1994) Efficacy of an influenza hemagglutinin-diphtheria toxoid conjugate vaccine in elderly nursing home subjects during an influenza outbreak. J Am Geriatr Soc 42:245-251. https://doi.org/10.1111/j.1532-5415.1994.tb01746.x

72. Goodwin K, Viboud C, Simonsen L (2006) Antibody response to influenza vaccination in the elderly: a quantitative review. Vaccine 24:1159-1169. https://doi.org/10.1016/j.vaccine.2005.08.105 
73. Ng A, Tam WW, Zhang MW, Ho CS, Husain SF, McIntyre RS, Hoe RC (2018) IL-1 $\beta$, IL-6, TNF- $\alpha$ and CRP in elderly patients with depression or Alzheimer's disease: systematic review and meta-analysis. Sci Rep. 8:12050. https://doi.org/10.1038/s41598-01830487-6

74. Anderson G, Maes M (2020) Gut dysbiosis dysregulates central and systemic homeostasis via suboptimal mitochondrial function: assessment, treatment and classification implications. Curr Top Med Chem 20:524-539. https://doi.org/10.2174/ 1568026620666200131094445

75. Vaduganathan M, Vardeny O, Michel T (2020) Renin-angiotensin-aldosterone system inhibitors in patients with Covid-19. N Engl J Med 382:1653-1659. https://doi.org/10.1056/nejmsr2005760

76. Wang LA, Kloet AD, Smeltzer MD, Cahill KM, Hiller H, Bruce EB, Pioquinto DJ, Ludin JA et al (2018) Coupling corticotropinreleasing-hormone and angiotensin converting enzyme 2 dampens stress responsiveness in male mice. Neuropharmacology 133:8593. https://doi.org/10.1016/j.neuropharm.2018.01.025

77. De Felice FG, Tovar-Moll F, Moll J, Munoz DP, Ferreira ST (2020) Severe acute respiratory syndrome coronavirus 2 (SARS-CoV-2) and the central nervous system. Trends Neurosci 43:355-357. https://doi.org/10.1016/j.tins.2020.04.004

78. Helmich RC, Bloem BR (2020) The impact of the COVID-19 pandemic on Parkinson's disease: hidden sorrows and emerging opportunities. J Park Dis 10:351-354. https://doi.org/10.3233/jpd202038

79. Hussain A, Bhowmik B, Moreira NCV (2020) COVID-19 and diabetes: knowledge in progress. Diabetes Res Clin Pract 9: 108142. https://doi.org/10.1016/j.diabres.2020.108142

80. Yao H, Chen JH, Xu YF (2020) Patients with mental health disorders in the COVID-19 epidemic. Lancet 7:21. https://doi.org/10. 1016/s2215-0366(20)30090-0

81. Ho CSH, Chee CY, Ho RCM (2020) Mental health strategies to combat the psychological impact of coronavirus disease 2019 (COVID-19). Beyond paranoia and panic. Ann Acad Med Singap 49:155-160 http://www.annals.edu.sg/pdf/49VolNo3Mar2020/ V49N3p155.pdf

82. World Health Organization (2020b) World Health Organization: mental health and psychosocial considerations during COVID-19 outbreak. https:/www.who.int/docs/default-source/coronaviruse/ mental-health-considerations.pdf.

83. Menon V (2020) Mental health among COVID-19 survivors: are we overlooking the biological links? Asian J Psychiatr 53:102217. https://doi.org/10.1016/j.ajp.2020.102217

84. Chen L, Deng H, Cui H, Fang J, Zuo Z, Deng J, Li Y, Wang X, Zhao L (2017) Inflammatory responses and inflammationassociated diseases in organs. Oncotarget 9:7204-7218. https:// doi.org/10.18632/oncotarget.23208

85. Ding Y, He L, Zhang Q, Huang Z, Che X, Hou J, Wang H, Shen H et al (2004) Organ distribution of severe acute respiratory syndrome (SARS) associated coronavirus (SARS-CoV) in SARS patients: implications for pathogenesis and virus transmission pathways. J Pathol 203:622-630. https://doi.org/10.1002/path.1560

86. Bhidayasiri R, Virameteekul S, Kim JM, Kr Pal P, Chung SJ (2020) COVID-19: an early review of its global impact and considerations for Parkinson's disease patient care. J Mov Disord 13:105-114. https://doi.org/10.14802/jmd.20042

87. Yang Y, Li W, Zhang Q, Zhang L, Cheung T, Xiang Y (2020) Mental health services for older adults in China during the COVID-19 outbreak. Lancet Psychiatry 7:19. https://doi.org/10. 1016/S2215-0366(20)30079-1

88. Li YC, Bai WZ, Hashikawa T (2020) The neuroinvasive potential of SARS-CoV2 may play a role in the respiratory failure of COVID-19 patients. J Med Virol 92:552-555. https://doi.org/10. 1002/jmv.25728

89. Chu H, Chan JFW, Yuen TTT, Shuai H, Yuan S, Wang Y, Hu B, Yip CCY et al (2020) Comparative tropism, replication kinetics, and cell damage profiling of SARS-CoV-2 and SARS-CoV with implications for clinical manifestations, transmissibility, and laboratory studies of COVID-19: an observational study. Lancet Microbe 1:14-23. https://doi.org/10.1016/S2666-5247(20)30004-5

90. Wu Y, Xu X, Chen Z, Duan J, Hashimoto K, Yang L, Liu C, Yang C (2020) Nervous system involvement after infection with COVID19 and other coronaviruses. Brain Behav Immun 87:18-22. https:// doi.org/10.1016/j.bbi.2020.03.031

91. Parma V, Ohla K, Veldhuizen MG, Niv MY, Kelly CE, Bakke AJ, Cooper KW, Bouysset C et al (2020) More than smell-COVID-19 is associated with severe impairment of smell, taste, and chemesthesis. Chem Senses 45:609-622. https://doi.org/10.1093/ chemse/bjaa041

92. Reger MA, Stanley IH, Joiner TE (2020) Suicide mortality and coronavirus disease 2019 - a perfect storm? JAMA Psychiatry 77(11):1093-1094. https://doi.org/10.1001/jamapsychiatry.2020. 1060

93. Lim WS, Liang CK, Assantachai P, Auyeung TW, Kang L, Lee WJ, Lim JY, Sugimoto K et al (2020) COVID-19 and older people in Asia: AWGS calls to actions. Geriatr Gerontol 20:547-558. https://doi.org/10.1111/ggi.13939

94. Jawaid A (2020) Protecting older adults during social distancing. Science 368:145-14145. https://doi.org/10.1126/science.abb7885

95. World Health Organization (2020c) Coronavirus. https://www. who.int/health-topics/coronavirus\#tab=tab_1. Accessed 11 Dec 2020

96. Koenig HG (2020) Ways of protecting religious older adults from the consequences of COVID-19. Am J Geriatr Psychiatry 28:776779. https://doi.org/10.1016/j.jagp.2020.04.004

97. Wang C, Pan R, Wan X, Tan Y, Xu L, Ho CS, Ho RC (2020b) Immediate psychological responses and associated factors during the initial stage of the 2019 coronavirus disease (COVID-19) epidemic among the general population in China. Int J Environ Res Public Health 17:1704-1729. https://doi.org/10.3390/ ijerph17051729

98. Centro Internacional de Longevidade Brasil (2015) Envelhecimento Ativo: Um Marco Político em Resposta à Revolução da Longevidade, first ed. Centro Internacional de Longevidade Brasil. Rio de Janeiro, Brasil

Publisher's Note Springer Nature remains neutral with regard to jurisdictional claims in published maps and institutional affiliations. 